\title{
Structure, electrical and gas sensor properties of Sn-Y-O thin films, prepared by reactive ion-beam sputtering
}

\author{
I. V. Babkina, O. V. Zhilova, Yu. Ye. Kalinin, V. A. Makagonov`, O. I. Remizova, A. V. Sitnikov \\ †vlad_makagonov@mail.ru
}

Voronezh State Technical University, 14 Moscovskii ave., Voronezh, 394026, Russia

\begin{abstract}
Experimental data on the structure, electrical and gas sensor properties of Sn-Y-O thin films with Y content from 0.4 to 4.8 at \% are presented. The films have been prepared by reactive ion-beam deposition in argon atmosphere with oxygen addition. Composite target used in deposition procedure represents a tin plate with yttrium strips unevenly arranged on the plate surface. Such a target configuration allowed one to obtain 75 samples with yttrium concentration 0.4 to 4.8 at. $\%$ in one deposition cycle. The structure of the $\mathrm{Sn}-\mathrm{Y}-\mathrm{O}$ films in the initial state is amorphous. The crystallization process in the system studied is observed at temperatures of $400-500^{\circ} \mathrm{C}$, and it begins with the formation of metastable $\mathrm{SnO}$ precipitates. The start temperature of the crystallization process increases with increasing of the $\mathrm{Y}$ content. Further increase of the temperature leads to a transformation of a part of the $\mathrm{SnO}$ phase into $\mathrm{SnO}_{2}$ and formation of crystalline $\mathrm{Y}_{2} \mathrm{O}_{3}$. The studies of electrical properties of the $\mathrm{Sn}$ - $\mathrm{Y}$-O thin films in the initial state show that electrical resistivity measured at room temperature is higher than $10^{10} \mathrm{Ohm} \cdot \mathrm{cm}$ and decreases with an increase of temperature. After heat treatment, leading to crystallization of Sn-Y-O thin films, electrotransport process at room temperatures is thermally activated with an activation energy about $0.23 \mathrm{eV}$. After crystallization the Sn-Y-O films show hydrogen gas-sensitivity properties. Particularly, the injection of hydrogen into argon at $350^{\circ} \mathrm{C}$ leads to a more than $60 \%$ decrease of electrical resistance for Sn-Y-O nanocrystalline thin film with Y concentration of 4.8 at. \%.
\end{abstract}

Keywords: tin oxide, conductivity, gas sensitivity.

УДК: 538.935, 539.216.2

\section{Структура, электрические и газосенсорные свойства тонких пленок Sn-Y-O, полученных методом реактивного ионно-лучевого напыления}

\author{
Бабкина И. В., Жилова О. В., Калинин Ю.Е., Макагонов В. А.†, Ремизова О. И., \\ Ситников А.В. \\ Воронежский государственный технический университет, Московский пр-т, 14, Воронеж, 394026, Россия
}

В работе рассмотрены результаты исследования структуры, электрических и газосенсорных свойств тонких пленок Sn-Y-O с содержанием Y от 0,4 до 4,8 ат.\%, полученных методом реактивного ионно-лучевого напыления в атмосфере аргона с добавлением кислорода. Составная мишень, используемая при напылении, представляет собой пластину олова с неравномерно расположенными на поверхности полосками иттрия. Такая конфигурация мишени позволила за один цикл напыления получить 75 образцов с концентрацией иттрия от 0,4 до 4,8 ат.\%. В исходном состоянии пленки Sn-Y-O имеют аморфную структуру. Процесс кристаллизации в полученной системе наблюдается при температурах $400-500^{\circ} \mathrm{C}$ и начинается с выделения метастабильной кристаллической фазы SnO. При этом температура начала процесса кристаллизации увеличивается с ростом содержания Ү. Дальнейшее увеличение температуры термообработки приводит к превращению части фазы $\mathrm{SnO}$ в $\mathrm{SnO}_{2}$ и выделению кристаллического соединения Ү $\mathrm{O}_{3}$. Исследования электрических свойств тонких пленок Sn-Y-O в исходном состоянии показали, что электрическое сопротивление при комнатной температуре имеет значение более $10^{10} \mathrm{OM} \cdot$ см и уменьшается с повышением температуры. После термообработки, приводящей к кристаллизации пленок Sn-Y-O, процесс электропереноса при температурах, близких к комнатной, является термоактивированным с энергией активации около 0,23 эВ. Пленки Sn-Y-O после кристаллизации проявляют газосенсорные свойства по отношению к водороду. В частности, введение водорода в среду аргона при температуре $350^{\circ} \mathrm{C}$ приводит к уменьшению электрического сопротивления нанокристаллической тонкой пленки Sn-Y-O с содержанием Y, равным 4,8 ат. \%, более чем на $60 \%$.

Ключевые слова: оксид олова, электропроводность, газовая чувствительность. 


\section{1. Введение}

Оксид олова $\left(\mathrm{SnO}_{2}\right)$ является типичным широкозонным полупроводником n-типа (3,8 эB) [1] и одним из наиболее широко используемых полупроводниковых оксидов. Легирование некоторыми элементами, такими как In, Sb и F, приводит к значительному увеличению электропроводности $\mathrm{SnO}_{2}$, он становится прозрачным для видимого света и отражает ИК-излучение. Благодаря своей химической стабильности и механической твердости, $\mathrm{SnO}_{2}$ является перспективным для применения в качестве прозрачных электродов для солнечных элементов, жидкокристаллических дисплеев, катализатора при доокислении метана, монооксидов углерода СО и азота NO, антистатических покрытий, газовых датчиков, анодов для литий-ионных батарей, прозрачных тонкопленочных транзисторов и т.д. [2-3].

Среди различных применений наиболее важным является применение $\mathrm{SnO}_{2}$ для газовых датчиков. Газочувствительные свойства датчиков на основе $\mathrm{SnO}_{2}$ (чувствительность, селективность и воспроизводимость) зависят от нескольких факторов, и в основном от размера кристаллитов и удельной поверхности. Например, газовая чувствительность начинает резко возрастать при уменьшении размера кристаллита (D) ниже критического значения (6 нм), что вдвое меньше толщины барьера Шоттки, проникающего внутрь кристаллита оксида олова. Весь кристаллит обеднен электронами, в результате чувствительность элемента к восстановительному газу будет увеличиваться [4-6]. Таким образом, использование наноструктурированных тонких пленок $\mathrm{SnO}_{2}$ может позволить получить улучшенные свойства твердотельных газовых датчиков.

Одним из перспективных направлений повышения функциональных свойств пленок $\mathrm{SnO}_{2}$ (уменьшение размера зерна, подавление процессов рекристаллизации при рабочих температурах, увеличение каталитической активности к различным газам, управление плотностью электронных состояний и подвижностью носителей заряда и т.д.) является поиск легирующих примесей. С этой точки зрения иттрий представляет особый интерес, так как является с одной стороны аморфизирующим элементом (что дает возможность создания нанокристаллической структуры за счет управляемой кристаллизации), с другой - акцепторной примесью, а также может выступать в качестве катализатора в процессе адсорбции водорода.

В данной работе рассмотрено влияние $\mathrm{Y}$ на фазовый состав, термическую стабильность электрических параметров и газосенсорных свойств тонких пленок Sn-Y-O, полученных методом реактивного ионно-лучевого напыления в атмосфере аргона с добавлением кислорода.

\section{2. Получение образцов и методика эксперимента}

Для получения тонких плёнок Sn-Y-О применялся метод реактивного ионно-лучевого распыления составной мишени [7], представляющей собой пластину олова разме-

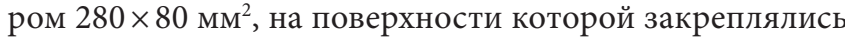

пять полосок иттрия размером $10 \times 80 \mathrm{mм}^{2}$ и толщиной 2 мм. Расстояние между навесками иттрия изменялось от 10 мм на одном краю мишени до 44 мм на другом. Такая компоновка составной мишени позволяла в одном технологическом процессе осаждения получать различные концентрации легирующего элемента, в нашем случае Y, в зависимости от положения подложки относительно мишени. В качестве подложек использовались пластины ситалла СТ-50-0,6.

В качестве основного рабочего газа использовался Ar чистотой 99.992\%. Выбор парциального давления реактивного газа $\mathrm{O}_{2}$ относительно $\mathrm{Ar}$ позволял получать пленки оксида олова с различным содержанием кислорода, что изменяло величину удельного электрического сопротивления пленок.

Элементный состав синтезированных тонких пленок определяли методом электронно-зондового микроанализа с помощью энергодисперсионной приставки INCA X-MAX (Oxford Instruments) к растровому электронному микроскопу Carl ZEISS EVO MA 10. Фазовый состав исследовали методом дифракции рентгеновских лучей на приборе Bruker D2 Phaser, обработку результатов осуществляли программными средствами Bruker DIFFRAC EVA 3.0 с применением базы данных ICDD PDF 2012 и TOPAS 4.2 .

Толщина полученных таким способом тонких пленок изменялась от 0,15 до 1,5 мкм, а содержание Y по - от 0,4 до 4,8 ат. \%.

\section{3. Структура пленок окислов олова, легированных иттрием}

Исследования структуры методом дифракции рентгеновских лучей показали, что в исходном состоянии пленки Sn-Y-O для изученных концентраций Y находятся в рентгеноаморфном состоянии (Рис. 1), а наблюдаемые максимумы на зависимости интенсивности дифрагированного рентгеновского пучка от двойного Брэгговского угла $\mathrm{I}(2 \Theta)$ принадлежат материалу подложки СТ-50-0,6. Для того, чтобы установить влияние температуры отжига на процесс кристаллизации и фазовый состав Sn-Y-O были проведены рентгенографические исследования пленок после термической обработки (Рис. 1). Выявлено, что кристаллизация пленки $\mathrm{Sn}-\mathrm{Y}-\mathrm{O}$ с 4 ат.\% Y происходит после термической обработки при $400^{\circ} \mathrm{C}$ в течение 30 минут (дифрактограмма 2 Рис. 1). Основной фазой после такой термообработки является тетрагональный оксид олова (II) SnO. Дифракционных максимумов, соответствующих другим соединениям, выявлено не было, однако на зависимости $\mathrm{I}(2 \Theta)$ сохранился широкий дифракционный максимум от рентгеноаморфной фазы.

Повышение температуры отжига приводит к образованию тетрагональной фазы $\mathrm{SnO}_{2}$ и кубического $\mathrm{Y}_{2} \mathrm{O}_{3}$ (дифрактограммы 3 и 4 Рис. 1). При этом на зависимости $\mathrm{I}(2 \Theta)$ площадь под широким гало от рентгеноаморфной фазы значительно уменьшается. Надо заметить, что при увеличении температуры термообработки с $500^{\circ} \mathrm{C}$ до $600^{\circ} \mathrm{C}$ происходит увеличение объемной доли фазы $\mathrm{SnO}_{2}$ и относительного уменьшения количества 


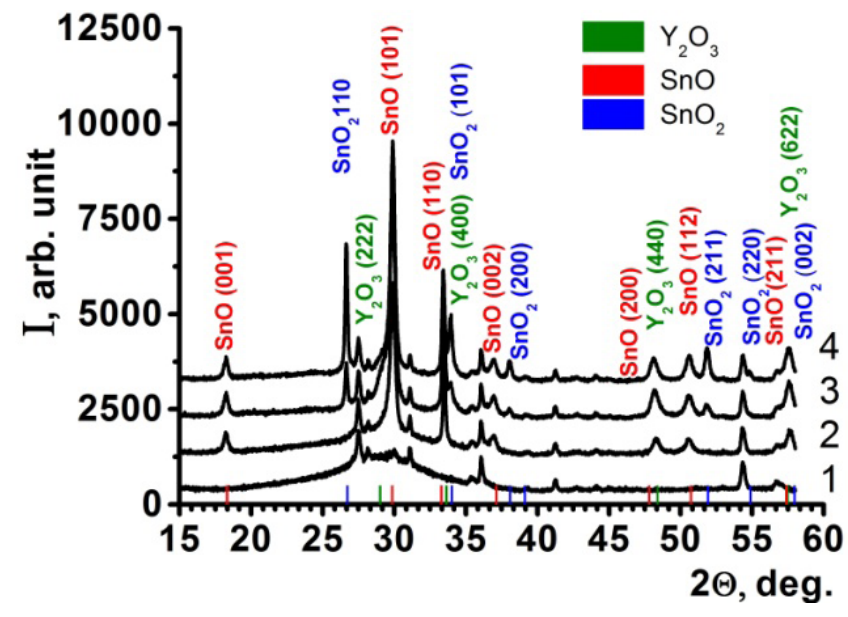

Pис. 1. (Color online) Дифрактограммы пленки Sn-Y-O (4 ат.\% Y) в исходном состоянии (1) после термической обработки в течение 30 минут при $400^{\circ} \mathrm{C}(2), 500^{\circ} \mathrm{C}$ (3) и $600^{\circ} \mathrm{C}(4)$.

Fig. 1. (Color online) Diffraction patterns of Sn-Y-O (4 at.\% Y) film in the initial state (1) and after heat treatment during 30 minutes at $400^{\circ} \mathrm{C}(2), 500^{\circ} \mathrm{C}(3)$ and $600^{\circ} \mathrm{C}(4)$.

$\mathrm{SnO}$ и $\mathrm{Y}_{2} \mathrm{O}_{3}$. Очевидно, фазы $\mathrm{SnO}_{2}$ и $\mathrm{Y}_{2} \mathrm{O}_{3}$ образуются на второй стадии кристаллизации пленок Sn-Y-O из рентгеноаморфной фазы.

Цепочку обнаруженных химических превращений, происходящих при термообработке пленок Sn-Y-O по результатам рентгенодифракционных исследований можно представить в виде:

$$
\begin{gathered}
\stackrel{\text { аморфная }}{\text { фаза }} \stackrel{400^{\circ} \mathrm{C}}{\longrightarrow} \mathrm{SnO}+\frac{\text { аморфная }}{\text { фаза }} \stackrel{500^{\circ} \mathrm{C}}{\longrightarrow} \\
\stackrel{500^{\circ} \mathrm{C}}{\longrightarrow} \mathrm{SnO}+\mathrm{SnO}_{2}+\mathrm{Y}_{2} \mathrm{O}_{3}+\frac{\text { аморфная }}{\text { фаза }} \downarrow \stackrel{600^{\circ} \mathrm{C}}{\longrightarrow} \\
\stackrel{600^{\circ} \mathrm{C}}{\longrightarrow} \mathrm{SnO} \downarrow+\mathrm{SnO}_{2} \uparrow+\mathrm{Y}_{2} \mathrm{O}_{3} \downarrow
\end{gathered}
$$

где стрелка «вверх» обозначает увеличение содержания той или иной фазы, а стрелка «вниз» - уменьшение.

\section{4. Электрические свойства пленок Sn-Y-O}

Описанные выше изменения фазового состава пленок Sn-Y-O непременно должны отражаться на процессах электропереноса исследуемых образцов. В связи с этим были исследованы температурные зависимости удельного электрического сопротивления пленок оксида олова с различным содержанием Ү (Рис. 2). В исходном состоянии при комнатной температуре все исследованные образцы имели высокое значение удельного электрического сопротивления $\left(\rho \approx 10^{10} \mathrm{Ohm} \times \mathrm{cm}\right)$. На зависимостях удельного электрического сопротивления от температуры $\rho(\mathrm{T})$, измеренных при нагреве (кривые 1 Рис. 2) в области температур $450-600^{\circ} \mathrm{C}$ наблюдается сильное уменьшение величины удельного электрического сопротивления пленок Sn-Y-O. При последующем охлаждении образца до комнатной температуры значение $\rho$ увеличивается, что характерно для полупроводниковых материалов.

Для объяснения зависимостей $\rho(\mathrm{T})$ обратимся к результатам исследования особенностей структуры полученных пленок Sn-Y-O после термической обработки:

- в исходном состоянии и до температуры $400^{\circ} \mathrm{C}$ тонкая пленка Sn-Y-O находится в рентгеноаморфном состоянии, характеризующимся большим значением удельного электрического сопротивления, вследствие закрепления уровня Ферми в середине запрещенной зоны полупроводника;

- при $400^{\circ} \mathrm{C}$ начинается процесс кристаллизации пленки с выделением кристаллитов SnO, но значительная часть материала остается в рентгеноаморфном состоянии. Удельное электрическое сопротивление начинает резко понижаться с ростом температуры;

- повышение температуры до $500^{\circ} \mathrm{C}$ приводит к уменьшению количества рентгеноаморфной фазы и появлению кристаллитов $\mathrm{SnO}_{2}$ и $\mathrm{Y}_{2} \mathrm{O}_{3}$;

- выше $600^{\circ} \mathrm{C}$ аморфная фаза полностью кристаллизуется с образованием трех оксидных соединений: $\mathrm{SnO}, \mathrm{SnO}_{2}$ и $\mathrm{Y}_{2} \mathrm{O}_{3}$.

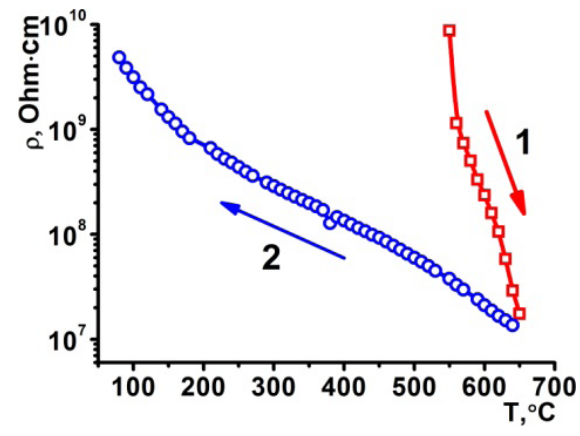

a

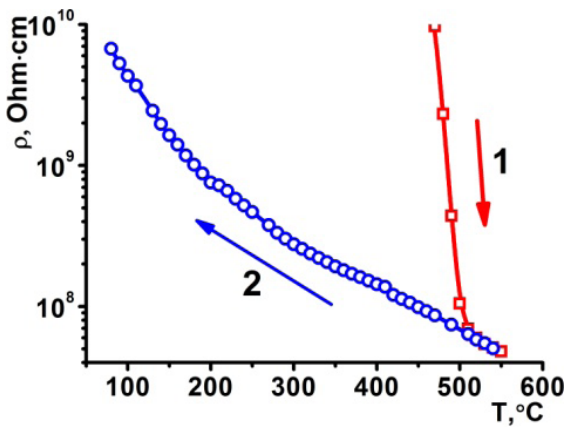

$\mathrm{b}$

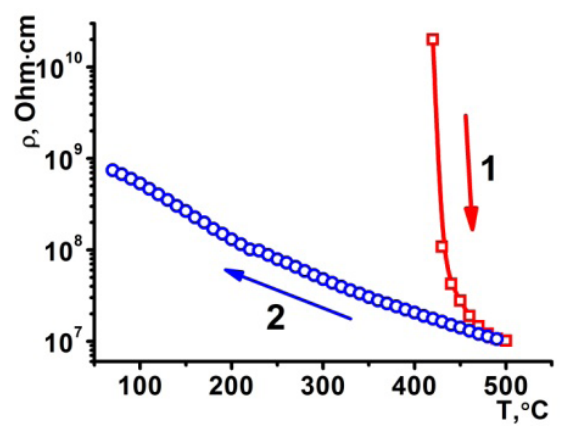

c

Рис. 2. Зависимости удельного электрического сопротивления от температуры пленок Sn-Y-O, измеренные в атмосфере аргона при $\mathrm{P}_{\mathrm{Ar}}=380$ Торр для пленок с различным содержанием Y: 2.2 ат. \% (a), 1.0 ат. \% (b), 0.4 ат. \% (c). Кривые 1 - измерение в режиме нагрева, кривые 2 - измерение в режиме охлаждения.

Fig. 2. Temperature dependencies of electrical resistivity of Sn-Y-O films measured in argon atmosphere at $\mathrm{P}_{\mathrm{Ar}}=380$ Torr for films with different $Y$ contents: 2.2 at. \% (a), 1.0 at. \% (b), 0.4 at. \% (c). Curves $1-$ measurement in heating mode, curves 2 - measurement in cooling mode. 
Таким образом, быстрое уменьшение величины удельного электрического сопротивления пленок $\mathrm{Sn}-\mathrm{Y}-\mathrm{O}$ в области температур $450-600^{\circ} \mathrm{C}$ связано с образованием бесконечной сетки соприкасающихся кристаллитов в аморфной матрице, при этом уменьшение содержания Y в пленке понижает температуру процесса кристаллообразования.

Зависимости удельного электрического сопротивления от температуры, измеренные в режиме охлаждения, могут быть аппроксимированы экспоненциальной зависимостью: $\rho \propto \exp \left(\mathrm{E}_{\mathrm{a}} / \mathrm{kT}\right)$, где $\mathrm{E}_{\mathrm{a}}-$ энергия активации проводимости. Поэтому, приведенные на Рис. 2 графики, были представлены в аррениусовских координатах: $\ln \rho-1 /$ T. Анализ рассчитанных значений энергии активации от концентрации Y показал, что в пределах погрешности величина составляет $\mathrm{E}_{\mathrm{a}} \approx 0,23 \pm 0,02$ эВ и может быть отнесена к примесному уровню атомов Y.

\section{5. Влияние водорода на электрические свойства тонких пленок Sn-Y-O}

Рассмотренные структурные превращения при термической обработке пленок Sn-Y-O, а также связанные с ними изменения электрических свойств должны оказывать влияние на процессы образования поверхностных состояний при адсорбции атомов различных газов. Для проверки этого предположения были проведены измерения удельного электросопротивления исследуемых пленок в среде воздуха и аргона с добавлением водорода. Измерения проводились на образцах предварительно подвергнутых термообработке при $600^{\circ} \mathrm{C}$ в течение 30 минут. В газовую среду (воздух или аргон), имеющую давление 380 Торр добавлялся водород с парциальным давлением 7,6 Topp.

За величину, характеризующую чувствительность полупроводникового резистивного элемента к различным газам удобно принять величину относительного изменения электрического сопротивления

$$
\mathrm{S}=\frac{\mathrm{R}}{\mathrm{R}_{0}} \cdot 100 \%,
$$

где $\mathrm{R}_{0}$ - равновесное значение электросопротивление элемента в газовой среде без водорода (воздух или аргон), R - значение электросопротивления элемента в момент измерения. В этом случае полученная величина характеризует степень изменения сопротивления в различных газовых средах.

Как уже было отмечено выше, Y может выступать в качестве катализатора в процессе адсорбции водорода и являться акцепторной примесью в позиции замещения атомов олова в пленках Sn-Y-O, поэтому образцы были исследованы как без дополнительного катализатора (Pd), так и с нанесением $\mathrm{Pd}$ из 5\% водного раствора $\mathrm{PdCl}_{2}$.

Было обнаружено, что нанесение каталитического Pd на поверхность пленки снижает величину относительного изменения электрического сопротивления пленок системы Sn-Y-O в водородосодержащей среде (см. Рис. 3).
Исследования газовой чувствительности пленок Sn-Y-O в другом диапазоне температур и концентраций Y показали, что добавление водорода может приводить к увеличению электрического сопротивления пленки (рис. 4).
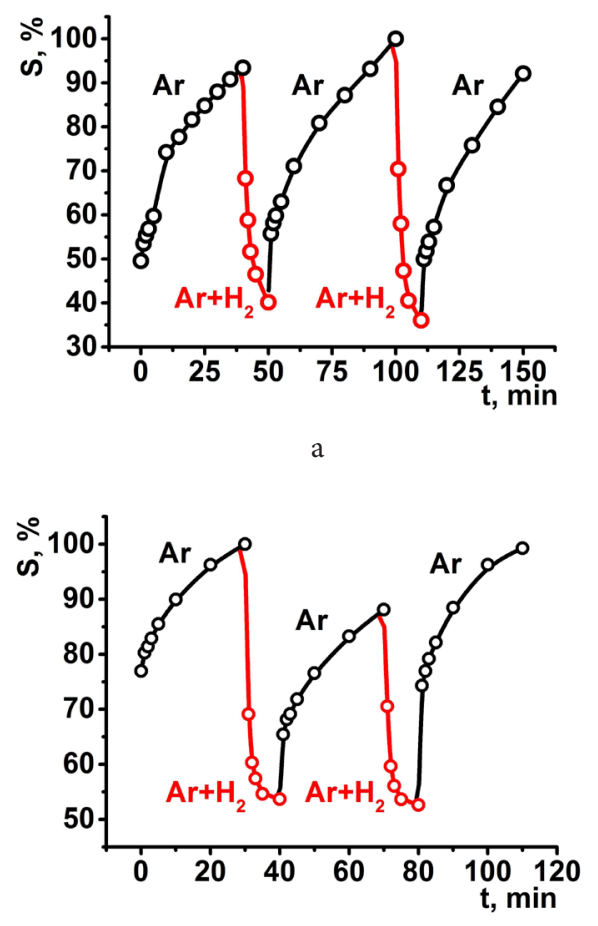

Рис. 3. Зависимости относительного изменения электрического сопротивления от времени для пленок системы Sn-Y-O с содержанием Y 4.8 ат. \% при напуске водорода в среду аргона при $\mathrm{T}=350^{\circ} \mathrm{C}$ : образец без $\mathrm{Pd}(\mathrm{a})$; образец с Pd (b).

Fig. 3. Time dependencies of the relative electrical resistance at $\mathrm{T}=350^{\circ} \mathrm{C}$ for $\mathrm{Sn}-\mathrm{Y}-\mathrm{O}$ films with $\mathrm{Y}$ content of 4.8 at. \% when hydrogen is injected into argon: sample without Pd (a); sample with Pd catalyst (b)

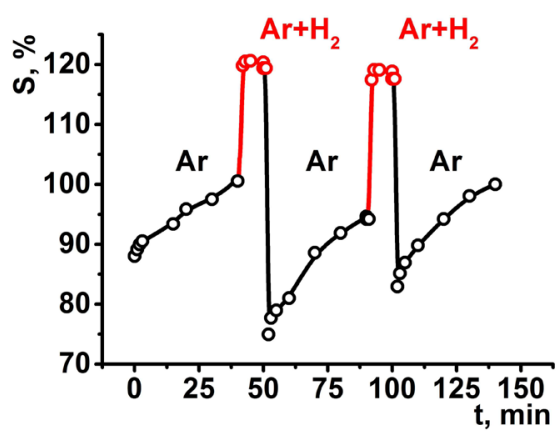

Рис. 4. Зависимость относительного изменения электрического сопротивления от времени для пленки системы Sn-Y-O с содержанием 2,2 ат. \% Y при напуске водорода в среду аргона при $\mathrm{T}=300^{\circ} \mathrm{C}$.

Fig. 4. Time dependencies of the relative electrical resistance at $\mathrm{T}=300^{\circ} \mathrm{C}$ for $\mathrm{Sn}-\mathrm{Y}-\mathrm{O}$ films with $\mathrm{Y}$ content of 2.2 at. \% when hydrogen is injected into argon. 
Обобщение полученных результатов представлено на Рис. 5, 6 и 7 и показывает, что положительный знак S проявляют пленки с концентрацией $\mathrm{Y} \sim 1$ ат. \% в области температур $200-350^{\circ} \mathrm{C}$ (Рис. 5 и 6). При этом величина $S$ максимальна при умеренных температурах $\sim 200^{\circ} \mathrm{C}$ (Рис. 7 кривая 2). Интересным фактом является то, что нанесение каталитического Pd на поверхность образца приводит к увеличению температурного диапазона, в котором наблюдается увеличение электрического сопротивления при добавлении водорода (Рис. 5).

Из литературы известно, что адсорбция водорода, независимо от типа проводимости полупроводника приводит к появлению в его приповерхностном слое до-

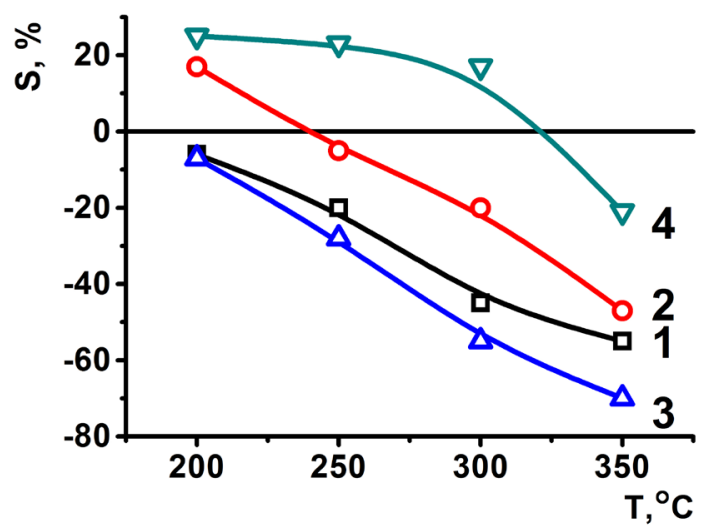

Рис. 5. Зависимости относительного изменения электрического сопротивления пленок Sn-Y-O от температуры при напуске водорода в среду аргона 1,3 - без нанесения катализатора (Pd) и 2,4 - поверхность образцов покрыта Pd, содержание Y: $1,2-4,8$ ат. \% и $3,4-2,2$ ат. \%.

Fig. 5. Temperature dependencies of the relative electrical resistance for $\mathrm{Sn}-\mathrm{Y}-\mathrm{O}$ films when hydrogen is injected into argon: 1,3 - without catalyst (Pd) and 2,4 - samples are coated by Pd, the $\mathrm{Y}$ content is $1,2-4.8$ at. $\%$ and $3,4-2.2$ at. \%.

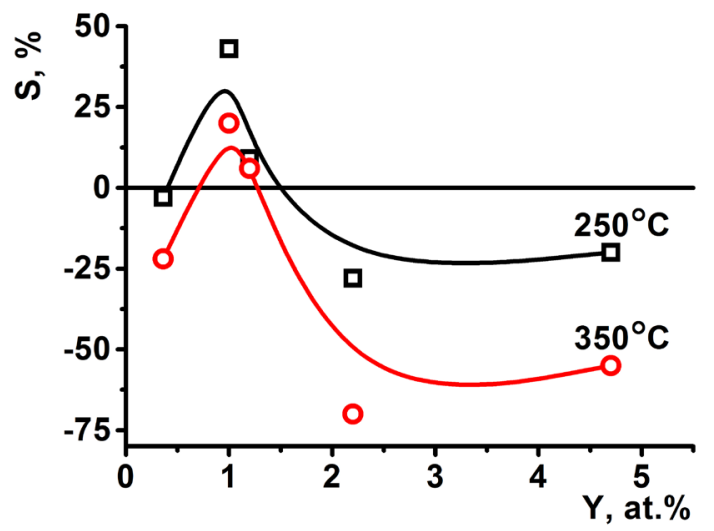

Рис. 6. Зависимости относительного изменения электрического сопротивления пленок Sn-Y-O от концентрации Y при напуске водорода в среду аргона при различной температуре без нанесения катализатора.

Fig. 6. Dependencies of the relative electrical resistance on $Y$ concentration for Sn-Y-O films without catalyst when hydrogen is injected into argon at different temperatures. норных состояний. Для полупроводников электронного типа это приводит к уменьшению удельного электрического сопротивления, а в полупроводниках дырочного типа удельное электрическое сопротивления увеличивается [8].

Чистый оксид олова $\mathrm{SnO}_{2}$ - полупроводник n-типа [9], и появление положительного S для пленок Sn-Y-O при добавлении водорода, позволяет сделать предположение, что после кристаллизации при невысоком содержании $\mathrm{Y} \leq 1$ ат. \% легирующий элемент занимает позиции олова к кристаллической решетке $\mathrm{SnO}_{2}$ и является акцептором. Нанесение каталитического Pd на поверхность образца приводит к увеличению степени адсорбции водорода, и, следовательно, к возрастанию степени компенсации приповерхностного слоя $\mathrm{SnO}_{2}$ и увеличению его удельного электрического сопротивления в газовой среде, содержащей водород. Увеличение доли Y в пленках Sn-Y-O приводит к сильному пересыщению $\mathrm{SnO}_{2}$ атомами Y, и в случае высокого содержания Y формируется фаза $\mathrm{Y}_{2} \mathrm{O}_{3}$. Как показано на рентгенограммах Рис. 1, в этом случае объемный $\mathrm{SnO}_{2}$ становится менее пересыщен иттрием, и степень компенсации приповерхностного слоя уменьшается. При этом пленки Y-Sn-O проявляют газосенсорные свойства, характерные для полупроводников n-типа.

\section{6. Заключение}

Пленки оксида олова, легированные 0,4-4,8 ат.\% Y, полученные методом реактивного ионно-лучевого напыления в исходном состоянии имеют аморфную структуру. Начало процесса кристаллизации в полученной системе наблюдается при температурах $400-500^{\circ} \mathrm{C}$, сопровождаясь выделением из рентгеноаморфной фазы метастабильной кристаллической фазы SnO. При этом температура начала кристаллизации увеличивается

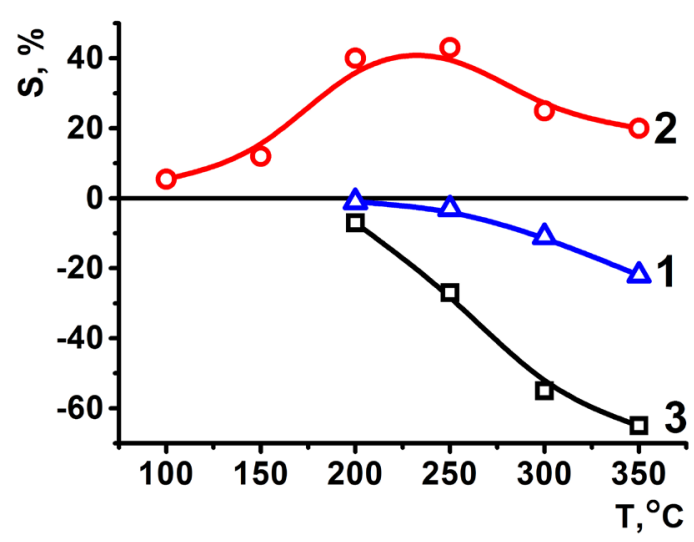

Рис. 7. Зависимости относительного изменения электрического сопротивления от температуры для пленок Sn-Y-O при напуске водорода в среду аргона без нанесения каталитического Pd. Содержание Y: 1 - 2.2 ат. \%, 2 - 1.0 ат. \%.3 - 0.4 ат. \%.

Fig. 7. Temperature dependencies of the relative electrical resistance for $\mathrm{Sn}$-Y-O films without catalyst $\mathrm{Pd}$ when hydrogen is injected into argon. $Y$ content is: $1-2.2$ at. $\%, 2-1.0$ at. $\%, 3-0.4$ at. $\%$. 
с ростом содержания Ү. Дальнейшее увеличение температуры приводит к превращению части фазы $\mathrm{SnO}$ в $\mathrm{SnO}_{2}$ и выделению кристаллического $\mathrm{Y}_{2} \mathrm{O}_{3}$. Выявлено, что после кристаллизации пленок оксида олова, легированного Y, процесс электропереноса при температурах близких к комнатной является термоактивационным с энергией активации $\mathrm{E}_{\mathrm{a}} \approx 0,23 \pm 0,02$ эВ. Для пленок $\mathrm{Sn}-\mathrm{Y}-\mathrm{O}$ значения $\mathrm{E}_{\mathrm{a}}$ можно связать с примесными уровнями, образованными растворенными атомами Y.

Пленки оксида олова с добавлением Ү от 0,4 до 4,8 ат. \% после кристаллизации проявляют газосенсорные свойства по отношению к водороду.

Обнаружено увеличение электрического сопротивления пленки Sn-Y-O с концентрацией Y $\approx 1$ ат. \% при добавлении водорода в инертную среду в температурном диапазоне $200-350^{\circ} \mathrm{C}$, что связано с тем, что Y в $\mathrm{SnO}_{2}$ проявляет акцепторные свойства.

Благодарность/Acknowledgements. Работа выполнена при поддержке Минобрнауки в рамках государственного задания (проект №3.1867.2017/4.6)./The work was supported by the Ministry of Education and Science within project part of the state task (project № 3.1867.2017/4.6).

\section{Литература/References}

1. J. Rockenberger, U. zum Felde, M. Tischer, L. Troger, M. Haase, H. Weller. J. Chem. Phys. 112, 4296 (2000). DOI: $10.1063 / 1.480975$
2. S. Monredon, A. Cellot, F. Ribot, C. Sanchez, L. Armelao, L. Gueneau, L. Delattre. J. Mater. Chem. 12, 2396 (2002). DOI: $10.1039 / \mathrm{b} 203049 \mathrm{~g}$

3. N.-S. Baik, G. Sakai, N. Miura, N. Yamazoe. J.Am. Ceram. Soc. 83, 2983 (2000). DOI: $10.1111 /$ j.1151-2916.2000.tb01670.x

4. H.-W.Ha, K. Kim, M. de Borniol, T. Toupance. J. Solid State Chem. 179, 702 (2006). DOI:10.1016/j.jssc.2005.11.022

5. E. S. Rembeza, O. Richard, J. V. Landuyt. Mater. Res. Bull. 34, 1527 (1999). DOI: 10.1016/S0025-5408(99)00188-9

6. N. Sergent, P. Gelin, L.P. Camby, H. Praliaud, G. Thomas. Sensors and Actuators B 84, 176 (2002). DOI: 10.1016/S0925-4005(02)00022-9

7. S.A. Gridnev, Yu.E. Kalinin, A.V. Sitnikov, O. V. Stognei. Nonlinear phenomena in nano- and microheterogeneous systems. Moscow, BINOM Laboratoriya znaniy (2012) 352 p. (in Russian) [С.А. Гриднев, Ю.Е. Калинин, А.В. Ситников, О.В. Стогней. Нелинейные явления в нано- и микрогетерогенных системах. Москва, БИНОМ Лаборатория знаний (2012) 352 с.]

8. Yu. A. Vashpanov, V.A. Smyntyna. Adsorption sensitivity of semiconductors. Odessa, «Astroprint» (2005) 216 p. (in Russian) [Ю.А. Вашпанов, В.А. Смынтына. Адсорбционная чувствительность полупроводников. Одесса, «Астропринт» (2005) 216 с.]

9. K.P. Bogdanov, D.T. Dimitrov, O.F. Lutskaya, Yu. M. Tairov. Semiconductors 32, 1033 (1998). DOI: $10.1134 / 1.1187560$ 